\title{
Oscillation criteria for solution to partial dynamic equations on time scales
}

\author{
R. Ramesh ${ }^{1}$ (D), Julio G. Dix ${ }^{2}$ (D), S. Harikrishnan ${ }^{3}$ (D), P. Prakash*4 (D) \\ ${ }^{1}$ Department of Mathematics, Muthayammal College of Engineering, Rasipuram - 637408, India \\ ${ }^{2}$ Department of Mathematics, Texas State University, 601 University Drive, San Marcos, TX 78666, USA \\ ${ }^{3}$ Department of Mathematics, Sona College of Technology, Salem - 636005, India \\ ${ }^{4}$ Department of Mathematics, Periyar University, Salem - 636011, India
}

\begin{abstract}
We consider the oscillatory behavior of solutions to partial dynamic equation on time scales. We establish several oscillation criteria by applying a Ricatti transformation. Examples are provided to justify our results.
\end{abstract}

Mathematics Subject Classification (2010). 35L10, 26E70, 34N05

Keywords. oscillation, partial dynamic equations, time scales

\section{Introduction}

Nowadays, the study of dynamic equations on time scale has received a lot of attention because of their applications in nuclear physics, control theory, engineering mechanics and other fields. The concept of time scales was introduced by Stephen Hilger in 1988 to unify discrete and continuous analysis. Bohner and Peterson [4] summarize the preliminaries and some applications of calculus on time scale; after that there has been more attention to research on time scales. Ahlbrandt and Morian [2] and Hoffacker [10] proposed the notion for multivariate cases and studied partial dynamic equations on time scales. Various definitions and extended ideas of the time scale calculus on the case of multivariate can be found in $[3,11]$.

Meanwhile oscillation properties of solutions plays a vital role in qualitative theory of difference and differential equations. Many authors have studied the oscillation for various types of equations such as differential and difference equation of integer and fractional order; see $[9,12,13,19]$. Also dynamic equation's oscillatory behavior on time scales has been studied in $[1,5-7,14,16-18]$. However, the oscillation of solutions of these type of partial dynamic equations on time scales were not considered earlier. This motivate the authors to establish the oscillation results of dynamic equations for multivariate cases.

We consider the partial dynamic equation

$$
\left(\alpha(t) y^{\Delta_{t}}(x, t)\right)^{\Delta_{t}}+p(x, t) y(x, t)=\beta(t) \nabla_{x}^{2} y(x, t), \quad(x, t) \in G \times\left[t_{0}, \infty\right)_{\mathbb{J}},
$$

\footnotetext{
${ }^{*}$ Corresponding Author.

Email addresses: pgramesh07@yahoo.co.in (R. Ramesh), jd01@txstate.edu (J.G. Dix), harikrish.10@gmail.com (S. Harikrishnan), pprakashmaths@gmail.com (P. Prakash)

Received: 15.04.2019; Accepted: 20.01.2020
} 
with either the Neumann boundary condition

$$
y_{N}(x, t)=0, \quad(x, t) \in \partial G \times\left[t_{0}, \infty\right)_{\mathbb{J}},
$$

or the Dirichlet boundary condition

$$
y(x, t)=0, \quad(x, t) \in \partial G \times\left[t_{0}, \infty\right)_{\mathbb{J}} .
$$

Here $G$ is a bounded domain in $\mathbb{R}^{n}$ with piecewise smooth boundary $\partial G, \Delta_{t}$ is the partial dynamic operator with respect to $t, \nabla_{x}^{2} y=\sum_{i=1}^{n} \partial_{x_{i} x_{i}}^{2} y$, and $N$ is the unit exterior normal vector to $\partial G$. The coefficients $\alpha(t)$ and $\beta(t)$ are rd continuous and real valued functions on $[0, \infty)_{\mathbb{J}}, \alpha(t)$ is positive and delta differentiable with $\alpha(t)^{\Delta_{t}}$ continuous, $p \in C(G \times$ $\left.\left[t_{0}, \infty\right)_{\mathbb{J}}, \mathbb{R}\right)$.

We establish sufficient conditions for the oscillation of (1.1)-(1.2), and of (1.1)-(1.3). We adapt to time scales the Ricatti transformation used for functional differential equations in $[15]$.

\section{Preliminaries}

The following definitions can be found in [4], where there is detailed introduction to time scale calculus. A time scale $\mathbb{J}$ is a nonempty closed subset of the real numbers $\mathbb{R}$. We will use intervals of the form $\left[t_{0}, \infty\right)_{\mathbb{J}}=\left[t_{0}, \infty\right) \cap \mathbb{J}$ for $t_{0} \in \mathbb{J}$. For a point $t \in \mathbb{J}$ we have following definitions: The forward jump operator is defined as $\sigma(t)=\inf \{s \in \mathbb{J}, s>t\}$. The backward jump operator is defined as $\rho(t)=\sup \{s \in \mathbb{J}, s<t\}$. The graininess is defined as $\mu(t)=\sigma(t)-t$. A point $t \in \mathbb{J}$ is said to be right-dense if $\sigma(t)=t$, and right-scattered if $\sigma(t)>t$.

A function $g: \mathbb{J} \rightarrow \mathbb{R}$ is said to be rd continuous if it is continuous at each right dense point and there exists a finite left limit of $g$ at all left dense points.

To define derivatives, we introduce

$$
\mathbb{J}^{\kappa}= \begin{cases}\mathbb{J} \backslash(\rho(\sup \mathbb{J}), \sup \mathbb{J}) & \text { if } \sup \mathbb{J}<\infty \\ \mathbb{J} & \text { if } \sup \mathbb{J}=\infty .\end{cases}
$$

At $t \in \mathbb{J}^{\kappa}$, the (delta) derivative of a function with respect to $t$ is the number $g^{\Delta}(t)=g^{\Delta_{t}}(t)$ (provided it exists) with the following property: For each $\epsilon>0$ there exists a $\delta$ positive, possibly depending on $t$, such that

$$
\left|(g(\sigma(t))-g(s))-g^{\Delta}(t)(\sigma(t)-s)\right| \leq \epsilon|\sigma(t)-s|
$$

for all $s \in(t-\delta, t+\delta) \cap \mathbb{J}$. A function $g: \mathbb{J} \rightarrow \mathbb{R}$ is said to be regressive provided $1+\mu(t) g(t) \neq 0$ for each $t \in \mathbb{J}^{k}$. Let $\mathcal{W}$ be the set of functions that are rd continuous and regressive. Also we define $\mathcal{W}^{+}=\{g \in \mathcal{W}: 1+\mu(t) g(t)>0, t \in \mathbb{J}\}$.

For $\gamma \in \mathcal{W}$ and $s, t \in \mathbb{J}$, the generalized exponential function is defined by

$$
e_{\gamma}(t, s)=\exp \left(\int_{s}^{t} \zeta_{\mu(\tau)}(\gamma(\tau)) \Delta \tau\right), \quad \zeta_{\mu}(z)= \begin{cases}\frac{1}{\mu} \log (1+\mu z), & \mu \neq 0 \\ z & \mu=0 .\end{cases}
$$

Then $e_{\gamma}(t, s)$ is a nonzero real valued function, and is the unique solution of the IVP

$$
v^{\Delta}(t)=\gamma(t) v(t), \quad v\left(t_{0}\right)=1 \quad \text { on } \mathbb{J} .
$$

Definition $2.1([11])$. Let $f: \mathbb{T}_{1} \times \mathbb{T}_{2} \times \ldots \times \mathbb{T}_{n} \rightarrow \mathbb{R}$ be a function and let $\mathbf{t}=$ $\left(t_{1}, t_{2}, \ldots, t_{i}, \ldots, t_{n}\right) \in \mathbb{T}^{k}$. Then define $f^{\Delta_{i}}(\mathbf{t})$ to be the number (provided it exists) with the property that given any $\epsilon>0$, there exists a neighborhood $U$ of $t_{i}$ with $U=$ $\left(t_{i}-\delta, t_{i}+\delta\right) \cap \mathbb{T}_{i}$ for $\delta>0$ such that

$\left|\left[f\left(t_{1}, \ldots \sigma\left(t_{i}\right), \ldots t_{n}\right)-f\left(t_{1}, \ldots s, \ldots t_{n}\right)\right]-f^{\Delta_{i}}(\mathbf{t})\left[\sigma\left(t_{i}\right)-s\right]\right| \leq \epsilon\left|\sigma_{i}(t)-s\right|$ for all $s \in U$.

$f^{\Delta_{i}}$ is called the partial delta derivative of $f$ at $\mathbf{t}$ with respect to the variable $t_{i}$. 
By a (classical) solution of (1.1), we mean a function $y(x, t): G \times\left[t_{0}, \infty\right)_{\mathbb{J}} \rightarrow \mathbb{R}$ such that $y, y^{\Delta_{t}},\left(y^{\Delta_{t}}\right)^{\Delta_{t}}, \partial_{x_{i}} y$, and $\partial_{x_{i} x_{j}}^{2} y$ are continuous on $\bar{G} \times\left[t_{0}, \infty\right)_{\mathbb{J}} \rightarrow \mathbb{R}$, for $i, j \in\{1, \ldots n\}$. Also the solution needs to satisfy (1.1) along with the boundary condition either (1.2) or (1.3).

Definition 2.2. The function $y(x, t)$ is said to be eventually positive (eventually negative) if $\exists t_{1} \geq t_{0}$ such that $y \geq 0$ and $\int_{G} y d x>0$ for all $t \geq t_{1}$ (resp. $y \leq 0$ and $\int_{G} y d x<0$ for all $\left.t \geq t_{1}\right)$.

There is also an alternative definition which is more restrictive. However our results hold under both definitions. A function $y(x, t)$ is called eventually positive (eventually negative) if $\exists t_{1} \geq t_{0}$ such that $y>0$ (resp. $y<0$ ) for all $t \geq t_{1}$ and all the interior points $x$ of $G$

A non-zero solution that is neither eventually positive nor eventually negative is called oscillatory; otherwise the solution is called non-oscillatory. Problem (1.1)-(1.2) or (1.1)(1.3) is called to be oscillatory if its every solution is oscillatory.

\section{Oscillation with Neumann boundary condition}

Assume that

$$
P(t):=\min _{x \in G} p(x, t) \geq 0 .
$$

Lemma 3.1. If $y(x, t)$ is an eventually positive solution of (1.1)-(1.2), then $\exists t_{1} \geq t_{0}$ so that $Y(t):=\int_{G} y d x>0$ and

$$
\left(\alpha(t) Y^{\Delta}(t)\right)^{\Delta}+P(t) Y(t) \leq 0, \quad \forall t \geq t_{1} .
$$

Also if $y(x, t)$ is an eventually negative solution, then $Y(t)<0$ and

$$
\left(\alpha(t) Y^{\Delta}(t)\right)^{\Delta}+P(t) Y(t) \geq 0, \quad \forall t \geq t_{1} .
$$

Proof. From the definition of eventually positive solution, there exist $t_{1} \geq t_{0}$ so that $Y>0$ for $t \geq t_{1}$. Then integrating (1.1) with respect to $x$ over $G$, we have

$$
\int_{G}\left(\alpha(t) y^{\Delta_{t}}(x, t)\right)^{\Delta_{t}} d x+\int_{G} p(x, t) y(x, t) d x=\beta(t) \int_{G} \nabla_{x}^{2} y(x, t) d x .
$$

Using Green's theorem and (1.2), we have

$$
\int_{G} \nabla_{x}^{2} y(x, t) d x=\int_{\partial G} \frac{\partial y(x, t)}{\partial N} d S=0
$$

Using that $\left(y^{\Delta_{t}}(x, t)\right)^{\Delta_{t}}$ is continuous with respect to $x$ on the bounded and closed subset $\bar{G} \subset \mathbb{R}^{n}$, we can obtain $\delta$ independent of $t$ in (2.1). Therefore the conditions in [4, Theorem 1.117] are satisfied, thus $\left(\int_{G} y(x, t) d x\right)^{\Delta_{t}}=\int_{G} y^{\Delta_{t}}(x, t) d x$. A similar arguments is used of the second derivative. From (3.4), (3.5), and (3.1), we obtain (3.2).

For eventually negative solutions, the proof follows the same process as above.

Lemma 3.2. Let $\int_{t_{0}}^{\infty} \frac{1}{\alpha(t)} \Delta t=\infty$ and $y$ be an eventually positive solution of (1.1)-(1.2). If $Y$ and $t_{1}$ are defined as in Lemma 3.1, then

$$
0 \leq Y^{\Delta}(s), \quad 0 \leq \frac{Y^{\Delta}(s)}{Y(s)} \leq \frac{1}{\alpha(s) \int_{t_{1}}^{s} \frac{1}{\alpha(\nu)} \Delta \nu} \quad \forall s \geq t_{1} .
$$

Proof. From (3.2), we have

$$
\left(\alpha(t) Y^{\Delta}(t)\right)^{\Delta} \leq-P(t) Y(t) \leq 0, \quad \forall t>t_{1} .
$$

Therefore $\alpha(t) Y^{\Delta}(t)$ is non-increasing; thus $\alpha(t) Y^{\Delta}(t)$ is of one sign. We assert that $\alpha Y^{\Delta} \geq 0$. To reach a contradiction, assume that there exists $t_{2} \geq t_{1}$ such that 
$c:=\alpha\left(t_{2}\right) Y^{\Delta}\left(t_{2}\right)<0$. Since $\alpha Y^{\Delta}$ is non-increasing, $Y^{\Delta}(s) \leq c / \alpha(s) \forall s \geq t_{2}$. Integrating from $t_{2}$ to $t$, we obtain

$$
Y(t)=Y\left(t_{2}\right)+\int_{t_{2}}^{t} Y^{\Delta}(s) \Delta s \leq Y\left(t_{2}\right)+c \int_{t_{2}}^{t} \frac{1}{\alpha(s)} \Delta s .
$$

Letting $t \rightarrow \infty$, the right-hand side approaches $-\infty$ which contradicts $Y$ being positive. Therefore $\alpha Y^{\Delta} \geq 0$. Dividing by $\alpha>0$ we obtain the left inequality in (3.6).

Since $Y$ is positive and $\alpha Y^{\Delta}$ is non-increasing,

$$
Y(t) \geq Y(t)-Y\left(t_{1}\right)=\int_{t_{1}}^{t} \frac{\alpha(\nu) Y^{\Delta}(\nu)}{\alpha(\nu)} \Delta \nu \geq \alpha(t) Y^{\Delta}(t) \int_{t_{1}}^{t} \frac{1}{\alpha(\nu)} \Delta \nu \quad \forall t>t_{1} .
$$

Dividing by $Y(t)>0$ and then by $\alpha(t) \int_{t_{0}}^{t} \frac{1}{\alpha(s)} \Delta s>0$, we obtain the right inequality in (3.6).

The next lemma considers eventually negative solutions; its proof follows the same steps as those in the above lemma.

Lemma 3.3. Let $\int_{t_{0}}^{\infty} \frac{1}{\alpha(t)} \Delta t=\infty$ and $y$ be an eventually negative solution of (1.1)-(1.2). If $Y$ and $t_{1}$ are defined as in Lemma 3.1, then

$$
Y^{\Delta}(s) \leq 0, \quad 0 \leq \frac{Y^{\Delta}(s)}{Y(s)} \leq \frac{1}{\alpha(s) \int_{t_{1}}^{s} \frac{1}{\alpha(\nu)} \Delta \nu} \quad \forall s \geq t_{1} .
$$

Theorem 3.4. Assume $\int_{t_{0}}^{\infty} \frac{1}{\alpha(t)} \Delta t=\infty$, and let

$$
I(t):=\frac{e_{\gamma}\left(\sigma(t), t_{0}\right) P(t)}{1+\frac{\mu(t)}{\alpha(t) \int_{t_{0}}^{s} \frac{1}{\alpha(\tau)} \Delta \tau}}-\frac{\alpha(t) e_{\gamma}^{2}\left(t, t_{0}\right) \gamma^{2}(t)}{4 e_{\gamma}\left(\sigma(t), t_{0}\right)}\left(1+\frac{\mu(t)}{\alpha(t) \int_{t_{0}}^{t} \frac{1}{\alpha(\tau)} \Delta \tau}\right) .
$$

If there exists an $\gamma \in \mathcal{W}^{+}$such that

$$
\limsup _{t \rightarrow \infty} \int_{t_{0}}^{t} I(s) \Delta s=\infty
$$

then (1.1)-(1.2) is oscillatory.

Proof. To reach a contradiction, initially we take that a solution $y$ is eventually positive. Let $Y$ and $t_{1}$ be defined as in Lemma 3.1, and using the Ricatti transformation, let

$$
w(t)=e_{\gamma}\left(t, t_{0}\right) \frac{\alpha(t) Y^{\Delta}(t)}{Y(t)} .
$$

By Lemmas 3.1 and 3.2, $Y>0$ and $Y^{\Delta} \geq 0$; therefore $w(t) \geq 0$ for $t \geq t_{1}$. Using the quotient rule, the product rule (see [4, Theorem 1.20]) and the derivative of $e_{\gamma}\left(t, t_{0}\right)$ we have

$$
\begin{aligned}
w^{\Delta}(t) & =e_{\gamma}^{\Delta}\left(t, t_{0}\right)\left(\frac{\alpha(t) Y^{\Delta}(t)}{Y(t)}\right)+e_{\gamma}\left(\sigma(t), t_{0}\right)\left(\frac{\alpha(t) Y^{\Delta}(t)}{Y(t)}\right)^{\Delta} \\
& =\gamma(t) w(t)+\frac{e_{\gamma}\left(\sigma(t), t_{0}\right)\left(\alpha(t) Y^{\Delta}(t)\right)^{\Delta}}{Y(\sigma(t))}-\frac{\alpha(t) e_{\gamma}\left(\sigma(t), t_{0}\right)\left(Y^{\Delta}(t)\right)^{2}}{Y(t) Y(\sigma(t))}
\end{aligned}
$$

From the definition of $w$, we have $Y^{\Delta}(t)=\frac{Y(t) w(t)}{\alpha(t) e_{\gamma}\left(t, t_{0}\right)}$. Then completing the square in the above expression we have

$$
\begin{aligned}
w^{\Delta}(t)= & -\frac{e_{\gamma}\left(\sigma(t), t_{0}\right) Y(t)}{\alpha(t) e_{\gamma}^{2}\left(t, t_{1}\right) Y(\sigma(t))} w^{2}(t)+\gamma(t) w(t)-\frac{\alpha(t) e_{\gamma}^{2}\left(t, t_{0}\right) Y(\sigma(t))}{4 e_{\gamma}\left(\sigma(t), t_{0}\right) Y(t)} \gamma^{2}(t) \\
& +\frac{\alpha(t) e_{\gamma}^{2}\left(t, t_{0}\right) Y(\sigma(t))}{4 e_{\gamma}\left(\sigma(t), t_{0}\right) Y(t)} \gamma^{2}(t)+\frac{e_{\gamma}\left(\sigma(t), t_{0}\right)\left(\alpha(t) Y^{\Delta}(t)\right)^{\Delta}}{Y(\sigma(t))}
\end{aligned}
$$


Using that the first three terms form the negative of a binomial squared, and (3.7), we have

$$
w^{\Delta}(t) \leq \frac{\alpha(t) e_{\gamma}^{2}\left(t, t_{0}\right) Y(\sigma(t))}{4 e_{\gamma}\left(\sigma(t), t_{0}\right) Y(t)} \gamma^{2}(t)-\frac{e_{\gamma}\left(\sigma(t), t_{0}\right) P(t) Y(t)}{Y(\sigma(t))} .
$$

By [4, Theorem 1.16(iv)] and (3.6), we have

$$
\frac{Y(\sigma(t))}{Y(t)}=1+\frac{\mu(t) Y^{\Delta}(t)}{Y(t)} \leq 1+\frac{\mu(t)}{\alpha(t) \int_{t_{0}}^{t} \frac{1}{\alpha(s)} \Delta s} .
$$

Then

$$
w^{\Delta}(t) \leq \frac{\alpha(t) e_{\gamma}^{2}\left(t, t_{0}\right) \gamma^{2}(t)}{4 e_{\gamma}\left(\sigma(t), t_{0}\right)}\left(1+\frac{\mu(t)}{\alpha(t) \int_{t_{0}}^{t} \frac{1}{\alpha(s)} \Delta s}\right)-\frac{e_{\gamma}\left(\sigma(t), t_{0}\right) P(t)}{1+\frac{\mu(t)}{\alpha(t) \int_{t_{0}}^{t} \frac{1}{\alpha(s)} \Delta s}}=-I(t),
$$

with $I(t)$ defined by (3.9). Taking integration from $t_{1}$ to $t$ yields

$$
w(t) \leq w\left(t_{1}\right)-\int_{t_{1}}^{t} I(s) \Delta s .
$$

Using that $I(s)$ is continuous on $\left[t_{0}, t_{1}\right]$, we get

$$
w(t) \leq w\left(t_{1}\right)+\int_{t_{0}}^{t_{1}} I(s) \Delta s-\int_{t_{0}}^{t} I(s) \Delta s .
$$

By (3.10) we obtain that $\lim _{t \rightarrow \infty} w(t)=-\infty$ which contradicts $w(t) \geq 0$. Therefore $y(x, t)$ can not be eventually positive.

Now we take that $y$ is eventually negative. By Lemma 3.3, $Y<0$ and $Y^{\Delta} \leq 0$, so that $w(t) \geq 0$. The same process as above yields a contradiction; thus $y(x, t)$ can not be eventually negative. Therefore $y(x, t)$ must be oscillatory.

Note that the conditions in Theorem 3.4 basically require $\alpha(t)$ not be too large, and $P(t)$ to be larger than $\alpha(t)$. In the next corollary we consider $\gamma=0$ that makes $e_{\gamma}\left(\sigma(s), t_{0}\right)=1$.

Corollary 3.5. Assume $\gamma(t)=0$ and $\int_{t_{0}}^{\infty} \frac{1}{\alpha(t)} \Delta t=\infty$. If

$$
\limsup _{t \rightarrow \infty} \int_{t_{0}}^{t} \frac{P(s)}{1+\frac{\mu(s)}{\alpha(s) \int_{t_{0}}^{s} \frac{1}{\alpha(\tau)} \Delta \tau}} \Delta s=\infty,
$$

then (1.1)-(1.2) is oscillatory.

Lemma 3.6. Let $\int_{t_{0}}^{\infty} \frac{1}{\alpha(t)} \int_{t_{0}}^{t} P(s) \Delta s \Delta t=\infty$ and $y$ be an eventually positive solution of (1.1)-(1.2). If $Y$ and $t_{1}$ be defined by Lemma 3.1, then either

$$
0 \leq Y^{\Delta}(s), \quad 0 \leq \frac{Y^{\Delta}(s)}{Y(s)} \leq \frac{1}{\alpha(s) \int_{t_{1}}^{s} \frac{1}{\alpha(\nu)} \Delta \nu} \quad \forall s \geq t_{1}
$$

or $\lim _{t \rightarrow \infty} Y(t)=0$.

Proof. As in Lemma 3.2, inequality (3.7) indicates that $\alpha(t) Y^{\Delta}(t)$ is non-increasing and is of one sign. Then two cases are available.

Case 1: $\alpha(t) Y^{\Delta}(t) \geq 0 \forall t \geq t_{1}$. Since $\alpha(t)>0$, it follows that $Y^{\Delta}(t) \geq 0$, which is the left inequality in (3.13). Since $Y(t)$ is positive and $\alpha(t) Y^{\Delta}(t)$ is non-increasing,

$$
Y(t) \geq Y(t)-Y\left(t_{1}\right)=\int_{t_{1}}^{t} \frac{\alpha(s) Y^{\Delta}(s)}{\alpha(s)} \Delta s \geq \alpha(t) Y^{\Delta}(t) \int_{t_{1}}^{t} \frac{1}{\alpha(s)} \Delta s \quad \forall t>t_{1} .
$$

Dividing by $Y(t)>0$ and then by $\alpha(t) \int_{t_{0}}^{t} \frac{1}{\alpha(s)} \Delta s>0$, we obtain the right inequality in (3.13). 
Case 2: $\alpha(t) Y^{\Delta}(t)<0 \forall t \geq t_{1}$. Since $\alpha(t) Y^{\Delta}(t)$ is non-increasing and $\alpha(t)$ is positive, $Y^{\Delta}(t)<0$ for all $t \geq t_{1}$. Then $Y(t)$ is positive and non-increasing, which implies that $\lim _{t \rightarrow \infty} Y(t)=: c \geq 0$. We claim that $c=0$. By contraction assuming that $c>0$, we have $Y(t) \geq c>0$ for all $t \geq t_{1}$. Then

$$
\left(\alpha(t) Y^{\Delta}(t)\right)^{\Delta}=-P(t) Y(t) \leq-c P(t) \quad \forall t \geq t_{1} .
$$

Integrating from $t_{1}$ to $t$, we have

$$
\alpha(t) Y^{\Delta}(t) \leq \alpha\left(t_{1}\right) Y^{\Delta}\left(t_{1}\right)-c \int_{t_{1}}^{t} P(s) \Delta s \leq-c \int_{t_{1}}^{t} P(s) \Delta s
$$

Dividing by $\alpha(t)>0$ and integrating again from $t_{1}$ to $t$, we have

$$
Y(t) \leq Y\left(t_{2}\right)-c \int_{t_{1}}^{t} \frac{1}{\alpha(s)} \int_{t_{1}}^{s} P(\tau) \Delta \tau \Delta s .
$$

Note the integrands are continuous on $\left[t_{0}, t_{1}\right]$, then from the assumption in this lemma, the right-hand side approaches $-\infty$ as $t \rightarrow \infty$. This contradicts $Y(t)$ being positive and implies $0=c=\lim _{t \rightarrow \infty} Y(t)$.

Lemma 3.7. Let $\int_{t_{0}}^{\infty} \frac{1}{\alpha(t)} \int_{t_{0}}^{t} P(s) \Delta s \Delta t=\infty$ and $y$ be an eventually negative solution of (1.1)-(1.2). If $Y$ and $t_{1}$ be defined by Lemma 3.1, then either

$$
Y^{\Delta}(s) \leq 0, \quad 0 \leq \frac{Y^{\Delta}(s)}{Y(s)} \leq \frac{1}{\alpha(s) \int_{t_{1}}^{s} \frac{1}{\alpha(\nu)} \Delta \nu} \quad \forall s \geq t_{1},
$$

or $\lim _{t \rightarrow \infty} Y(t)=0$.

The proof is identical to that of Lemma 3.6, we omit it.

Theorem 3.8. Assume $\int_{t_{0}}^{\infty} \frac{1}{\alpha(t)} \int_{t_{0}}^{t} P(s) \Delta s \Delta t=\infty$ and let $I(t)$ be as defined in Theorem 3.4. If there exists an $\gamma \in \mathcal{W}^{+}$such that $\lim _{\sup _{t \rightarrow \infty}} \int_{t_{0}}^{t} I(s) \Delta s=\infty$ then every solution of (1.1)-(1.2) is oscillatory or tends to zero as $t \rightarrow \infty$.

Proof. Initially assume that the solution is eventually positive. Then we consider the two cases in Lemma 3.6. For case 1 we proceed as in Theorem 3.4. For case 2 we also proceed as in Lemma 3.6.

Now assume the solution is eventually negative. We consider Lemma 3.7 in the two possible cases and obtain the desired conclusion.

\section{Oscillation with Dirichlet boundary condition}

In this section, we use the eigenvalues of the Laplacian,

$$
\begin{gathered}
-\nabla^{2} \phi(x)=\lambda \phi(x) \text { in } G, \\
\phi(x)=0 \text { on } \partial G .
\end{gathered}
$$

The principal eigenvalue $\lambda_{1}$ is positive and the corresponding eigenfunction $\phi_{1}$ is also positive in the interior of $G$; see [8, Theorem 2, page 356]. Furthermore, we normalize this eigenvector so that $\int_{G} \phi_{1}=1$. We shall assume that

$$
P+\lambda_{1} \beta \geq 0, \quad \text { where } P(t)=\min _{x \in G} p(x, t) .
$$

Lemma 4.1. If $y(x, t)$ is an eventually positive solution of (1.1)-(1.3), then $\exists t_{1} \geq t_{0}$ such that $Y(t):=\int_{G} y(x, t) \phi_{1}(x) d x>0$ and

$$
\left(\alpha(t) Y^{\Delta}(t)\right)^{\Delta}+\left(P(t)+\lambda_{1} \beta(t)\right) Y(t) \leq 0, \quad \forall t \geq t_{1} .
$$

Also if $y(x, t)$ is an eventually negative solution, then $Y(t)<0$ and

$$
\left(\alpha(t) Y^{\Delta}(t)\right)^{\Delta}+\left(P(t)+\lambda_{1} \beta(t)\right) Y(t) \geq 0, \quad \forall t \geq t_{1} .
$$


Proof. From the definition of eventually positive solution, there exist $t_{1} \geq t_{0}$ such that $y(x, t) \geq 0$ and $\int_{G} y(x, t) d x>0$ for $t \geq t_{1}$. Since $\phi_{1}(x)>0$ in the interior of $G$, it follows that $Y(t)=\int_{G} y(x, t) \phi_{1}(x) d x>0$. We multiply (1.1) by $\phi_{1}$ and integrate with respect to $x$ over $G$ and get

$$
\begin{aligned}
& \int_{G}\left(\alpha(t) y^{\Delta_{t}}(x, t)\right)^{\Delta_{t}} \phi_{1}(x) d x+\int_{G} p(x, t) y(x, t) \phi_{1}(x) d x \\
& =\beta(t) \int_{G} \nabla_{x}^{2} y(x, t) \phi_{1}(x) d x .
\end{aligned}
$$

On the right-hand side, we have

$$
\beta(t) \int_{G} \nabla^{2} y(x, t) \phi_{1}(x) d x=-\lambda_{1} \beta(t) \int_{G} y(x, t) \phi_{1}(x) d x .
$$

As in Lemma 3.1, by [4, Theorem 1.117], we have $\left(\int_{G} y(x, t) d x\right)^{\Delta_{t}}=\int_{G} y^{\Delta_{t}}(x, t) d x$, and a similar statement for the second derivative. From (4.4), (4.5), and (4.1), we obtain (4.2).

For eventually negative solutions, the proof follows the same process as above.

Lemma 4.2. Let $\int_{t_{0}}^{\infty} \frac{1}{\alpha(t)} \Delta t=\infty$ and $y$ be an eventually positive solution of (1.1)-(1.3). If $Y$ and $t_{1}$ be taken as in Lemma 4.1, then

$$
0 \leq Y^{\Delta}(s), \quad 0 \leq \frac{Y^{\Delta}(s)}{Y(s)} \leq \frac{1}{\alpha(s) \int_{t_{1}}^{s} \frac{1}{\alpha(\nu)} \Delta \nu} \quad \forall s \geq t_{1} .
$$

Proof. From (4.2), we have

$$
\left(\alpha Y^{\Delta}\right)^{\Delta} \leq-\left(P+\lambda_{1} \beta\right) Y \leq 0, \quad \forall t>t_{1} .
$$

Then the rest of the proof is the same as that in Lemma 3.2.

The next lemma considers eventually negative solutions; its proof follows the same steps as those in the above lemma.

Lemma 4.3. Let $\int_{t_{0}}^{\infty} \frac{1}{\alpha(t)} \Delta t=\infty$ and $y$ be an eventually negative solution of (1.1)-(1.3). If $Y$ and $t_{1}$ be taken as in Lemma 4.1, then

$$
Y^{\Delta}(s) \leq 0, \quad 0 \leq \frac{Y^{\Delta}(s)}{Y(s)} \leq \frac{1}{\alpha(s) \int_{t_{1}}^{s} \frac{1}{\alpha(\nu)} \Delta \nu} \quad \forall s \geq t_{1} .
$$

Theorem 4.4. Assume (4.1) and $\int_{t_{0}}^{\infty} \frac{1}{\alpha(t)} \Delta t=\infty$, and let

$$
J(t):=\frac{e_{\gamma}\left(\sigma(t), t_{0}\right)\left(P(t)+\lambda_{1} \beta(t)\right)}{1+\frac{\mu(t)}{\alpha(t) \int_{t_{0}}^{t} \frac{1}{\alpha(\tau)} \Delta \tau}} \Delta s-\frac{\alpha(t) e_{\gamma}^{2}\left(s, t_{0}\right) \gamma^{2}(t)}{4 e_{\gamma}\left(\sigma(t), t_{0}\right)}\left(1+\frac{\mu(t)}{\alpha(t) \int_{t_{0}}^{t} \frac{1}{\alpha(\tau)} \Delta \tau}\right) .
$$

If there exists an $\gamma \in \mathcal{W}^{+}$such that $\lim \sup _{t \rightarrow \infty} \int_{t_{0}}^{t} J(s) \Delta s=\infty$ then (1.1)-(1.3) is oscillatory.

The proof of the above theorem is identical to that of Theorem 3.4, substituting $P$ by $P+\lambda_{1} \beta$

Corollary 4.5. Assume $\gamma(t)=0$ and $\int_{t_{0}}^{\infty} \frac{1}{\alpha(t)} \Delta t=\infty$. If

$$
\limsup _{t \rightarrow \infty} \int_{t_{0}}^{t} \frac{P(s)+\lambda_{1} \beta(t)}{1+\frac{\mu(s)}{\alpha(s) \int_{t_{0}}^{s} \frac{1}{\alpha(\tau)} \Delta \tau}} \Delta s=\infty,
$$

then (1.1)-(1.3) is oscillatory. 
Lemma 4.6. Assume that

$$
\int_{t_{0}}^{\infty} \frac{1}{\alpha(t)} \int_{t_{0}}^{t}\left(P(s)+\lambda_{1} \beta(s)\right) \Delta s \Delta t=\infty
$$

and $y$ is an eventually positive solution of (1.1)-(1.3). If $Y$ and $t_{1}$ be taken by Lemma 4.1, then either

$$
0 \leq Y^{\Delta}(s), \quad 0 \leq \frac{Y^{\Delta}(s)}{Y(s)} \leq \frac{1}{\alpha(s) \int_{t_{1}}^{s} \frac{1}{\alpha(\nu)} \Delta \nu} \quad \forall s \geq t_{1},
$$

or $\lim _{t \rightarrow \infty} Y(t)=0$.

The proof is same as that of Lemma 3.6.

Lemma 4.7. Assume that

$$
\int_{t_{0}}^{\infty} \frac{1}{\alpha(t)} \int_{t_{0}}^{t}\left(P(s)+\lambda_{1} \beta(s)\right) \Delta s \Delta t=\infty
$$

and $y$ is an eventually negative solution of (1.1)-(1.3). If $Y$ and $t_{1}$ be taken by Lemma 4.1, then either

$$
Y^{\Delta}(s) \leq 0, \quad 0 \leq \frac{Y^{\Delta}(s)}{Y(s)} \leq \frac{1}{\alpha(s) \int_{t_{1}}^{s} \frac{1}{\alpha(\nu)} \Delta \nu} \quad \forall s \geq t_{1}
$$

or $\lim _{t \rightarrow \infty} Y(t)=0$.

The proof is identical to that of Lemma 4.6, we omit it.

Theorem 4.8. Assume

$$
\int_{t_{0}}^{\infty} \frac{1}{\alpha(t)} \int_{t_{0}}^{t}\left(P(s)+\lambda_{1} \beta(s)\right) \Delta s \Delta t=\infty
$$

and let $J(t)$ be as defined in Theorem 4.4. If there exists an $\gamma \in \mathcal{W}^{+}$such that $\limsup _{t \rightarrow \infty} \int_{t_{0}}^{t} J(s) \Delta s=\infty$ then (1.1)-(1.3) is oscillatory or tends to zero as $t \rightarrow \infty$.

Proof. Initially take that the solution is eventually positive. Then we consider the two cases in Lemma 4.6. For case 1 we proceed as in Theorem 3.4. For case 2 we also proceed as in Lemma 4.6.

Now assume the solution is eventually negative. We consider Lemma 4.7 in the two possible cases and obtain the desired conclusion.

\section{Examples}

Example 5.1. Consider

$$
\begin{gathered}
\left(\frac{1}{t} y^{\Delta_{t}}\right)^{\Delta_{t}}+t^{2} y=t \nabla_{x}^{2} y, \quad(x, t) \in(0, \pi) \times[1, \infty)_{\mathbb{J}}, \\
y_{x}(0, t)=y_{x}(\pi, t)=0, \quad t \in[1, \infty)_{\mathbb{J}} .
\end{gathered}
$$

Here $\alpha(t)=1 / t, \beta(t)=t, p(x, t)=t^{2}, P(t)=t^{2}$.

Let $\mathbb{J}=\left\{q^{n}: q>1, n \in \mathbb{N} \cup\{0\}\right\}$ and $\gamma(t)=0$. Then

$$
\int_{t_{0}}^{\infty} \frac{1}{\alpha(t)} \Delta t=\lim _{t \rightarrow \infty} \int_{1}^{t} s \Delta s=\lim _{t \rightarrow \infty} \frac{t^{2}-1}{1+q}=\infty .
$$

and

$$
\limsup _{t \rightarrow \infty} \int_{t_{0}}^{t} \frac{P(s)}{1+\frac{\mu(s)}{\alpha(s) \int_{t_{0}}^{s} \frac{1}{\alpha(\tau)} \Delta \tau}} \Delta s=\limsup _{t \rightarrow \infty} \int_{1}^{t} \frac{s^{2}\left(s^{2}-1\right)}{q^{2} s^{2}-1} \Delta s=\infty .
$$

The conditions of Corollary 3.5 hold. Therefore (5.1) is oscillatory. 
Example 5.2. Consider

$$
\begin{gathered}
\left(t y^{\Delta_{t}}\right)^{\Delta_{t}}+t^{2} y=t^{2} \nabla_{x}^{2} y,(x, t) \in(0, \pi) \times[1, \infty)_{\mathbb{J}}, \\
y(0, t)=y(\pi, t)=0, \quad t \in[1, \infty)_{\mathbb{J}} .
\end{gathered}
$$

Here $\alpha(t)=t, \beta(t)=t^{2}, p(x, t)=t^{2}, P(t)=t^{2}$. The principal eigenvalue is $\lambda_{1}=1$ and $\phi_{1}=\sin (x) / 2$. Let $\mathbb{J}=\left\{q^{n}: q>1, n \in \mathbb{N} \cup\{0\}\right\}$ and $\gamma(t)=0$. Then

$$
\int_{t_{0}}^{\infty} \frac{1}{\alpha(t)} \int_{t_{0}}^{t}\left(P(s)+\lambda_{1} \beta(s)\right) \Delta s \Delta t=\lim _{t \rightarrow \infty} \int_{1}^{t} \frac{1}{s}\left(\frac{2\left(s^{3}-1\right)}{1+q+q^{2}}\right) \Delta s=\infty .
$$

and

$$
\begin{aligned}
\limsup _{t \rightarrow \infty} \int_{t_{0}}^{t} J(s) \Delta s & =\limsup _{t \rightarrow \infty} \int_{t_{0}}^{t} \frac{P(s)+\lambda_{1} \beta(s)}{1+\frac{\mu(s)}{\alpha(s) \int_{t_{0}}^{s} \frac{1}{\alpha(\tau)} \Delta \tau}} \Delta s \\
& =\limsup _{t \rightarrow \infty} \int_{1}^{t} 2 s^{2} \log (s-q s) \Delta s=\infty
\end{aligned}
$$

The conditions of Theorem 4.8 are satisfied. Therefore (5.2) is oscillatory.

Acknowledgment. The fourth author was supported by Department of Science and Technology, New Delhi, INDIA under FIST programme SR/FST/MS1-115/2016.

\section{References}

[1] R.P. Agarwal, M. Bohner and S.H. Saker, Oscillation of second order delay dynamic equations, Can. Appl. Math. Q. 13, 1-18, 2005.

[2] C.D. Ahlbrandt and C. Morian, Partial differential equations on time scales, J. Comput. Appl. Math. 141, 35-55, 2002.

[3] M. Bohner and G.S. Guseinov, Partial differentiation on time scales, Dynam. Systems Appl. 13, 351-379, 2004.

[4] M. Bohner and A. Peterson, Dynamic Equations on Time Scale, An Introduction with Applications, Birkhäuser, Boston, 2001.

[5] M. Bohner and S.H. Saker, Oscillation of second order nonlinear dynamic equations on time scales, Rocky Mountain J. Math. 34, 1239-1254, 2004.

[6] M. Bohner, L. Erbe and A. Peterson, Oscillation for nonlinear second order dynamic equations on time scales, J. Math. Anal. Appl. 301, 491-507, 2005.

[7] L. Erbe, A. Peterson and S.H. Saker, Oscillation criteria for second order nonlinear dynamic equations on time scales, J. Lond. Math. Soc. 67 (3), 701-714, 2003.

[8] L.C. Evans, Partail Differential Equations, American Math. Society, Graduate Studies in Mathematics Vol. 19, second ed., 2010.

[9] P. Hasil and M. Veselý, Oscillation and nonoscillation criteria for linear and half linear difference equations, J. Math. Anal. Appl. 452 (1), 401-428, 2017.

[10] J. Hoffacker, Basic partial dynamic equations on time scales, J. Difference Equ. Appl. 8 (4), 307-319, 2002.

[11] B. Jackson, Partial dynamic equations on time scales, J. Comput. Appl. Math. 186 (2), 391-415, 2006.

[12] P. Prakash and S. Harikrishnan, Oscillation of solutions of impulsive vector hyperbolic differential equations with delays, Appl. Anal. 91 (3), 459-473, 2012.

[13] P. Prakash, S. Harikrishnan and M. Benchohra, Oscillation of certain nonlinear fractional partial differential equation with damping term, Appl. Math. Lett. 43, 72-79, 2015.

[14] S.H. Saker, Oscillation criteria of second order half linear dynamic equations on time scales, J. Comput. Appl. Math. 177 (2), 375-387, 2005. 
[15] Y. Shoukaku and N. Yoshida, Osillations of nonlinear hyperbolic equations with functional arguments via Riccati method, Appl. Math. Comput. 217, 143-151, 2010.

[16] S. Sun, Z. Han and C. Zhang, Oscillation of second order delay dynamic equations on time scales, J. Appl. Math. Comput. 30 (1-2), 459-468, 2009.

[17] Q. Zhang, Oscillation of second order half linear delay dynamic equations with damping on time scales, J. Comput. Appl. Math. 235 (5), 1180-1188, 2011.

[18] Q. Zhang and L. Gao, Oscillation of second order nonlinear delay dynamic equations with damping on time scales, J. Appl. Math. Comput. 37 (1-2), 145-158, 2011.

[19] X. Zhou, C. Liu and W.-S. Wang, Interval oscillation criteria for nonlinear differential equations with impulses and variable delay, Appl. Math. Lett. 85, 150-156, 2018. 